\title{
Use of oral sodium cromoglycate in persistent diarrhoea
}

\author{
T D BOLIN* \\ From the University of New South Wales, Gastrointestinal Unit, Prince of Wales Hospital, Randwick, \\ New South Wales, Australia.
}

SUMMARY Twenty patients with persistent diarrhoea participated in a randomised, double-blind trial of oral sodium cromoglycate and placebo. Eight patients noted significant improvement in their diarrhoea while taking sodium cromoglycate and this did not correlate with the presence of other atopic diseases, a history of food intolerance, or the presence of lactase deficiency. The results suggest that some patients with diarrhoea of unknown cause may have food allergy as a major contributing cause for their diarrhoea.

It is usually accepted that $50 \%$ of patients presenting to a gastrointestinal clinic with chronic diarrhoea have no organic cause for their symptoms. ${ }^{1}$ This situation is virtually unique when the aetiology of other important non-gastrointestinal symptoms is considered and may well reflect our poor understanding of the pathogenesis of some gastrointestinal symptoms.

The concept that food allergy can provoke a variety of diverse symptoms has recently received increasing support. There is very little evidence that gastrointestinal symptoms, such as diarrhoea have their basis in allergy to certain foods if conditions such as gluten sensitive enteropathy, cows milk, and soya protein intolerance are excluded.

There is, however, recent evidence that treatment with oral sodium cromoglycate protects against the development of asthma and eczema after an oral allergen challenge. ${ }^{23}$ It was therefore decided to examine the possibility that some patients with persistent diarrhoea without obvious cause (irritable bowel syndrome) may be symptomatically improved with oral SCG. If this was beneficial it would lend some support to the concept that food allergy may play a role in certain patients with unexplained diarrhoea.

\section{Methods}

PATIENTS

Twenty patients were chosen from a group of 100 patients participating in a prospective study of

*Address for correspondence: Gastrointestinal Unit, Old Stone Building, South Wing, Prince of Wales Hospital, Randwick, 2031, New South Wales, Australia.

Received for publication 13 May 1980 persistent diarrhoea, when a final diagnosis of irritable bowel syndrome had been made. Persistent diarrhoea was defined as a change in bowel habit, resulting in alteration of the consistency of stools, in that they became sloppy or liquid. Investigation of their diarrhoea had included a full blood count, biochemical profile, stool microscopy and culture, sigmoidoscopy, barium enema, small bowel series and a small bowel biopsy with disaccharidase assay and examination of duodenal juice for Giardia lamblia trophozoites. Furthermore, each patient had been given an empirical therapeutic trial of either metronidazole (400 mg eight hourly for seven days) or tinidazole ( $2 \mathrm{~g}$ statim; repeated in 48 hours) to exclude occult giardiasis as the cause of their diarrhoea. Symptoms had been present for a mean of 14 years (range three to 30 years). In no patient was there a history of intolerance to milk or any obvious specific food intolerance; 12 patients, however, described a general intolerance to a wide variety of foods without being able to specify any that precipitated diarrhoea. A personal and family history of allergic and atopic disease was obtained. No patient was on medication which might cause diarrhoea.

Patients entered into the trial began by recording, on a chart, their symptoms, particularly in relation to frequency and consistency of bowel motions, abdominal pain, and bloating. After four weeks they were randomly allocated to treatment with either oral SCG or placebo for a four week period, given on a double-blind basis. At the end of four weeks they changed to the alternative treatment. Daily recording of symptom severity continued throughout each treatment period. An assessment of symptom 
Table Response to treatment with sodium cromoglycate

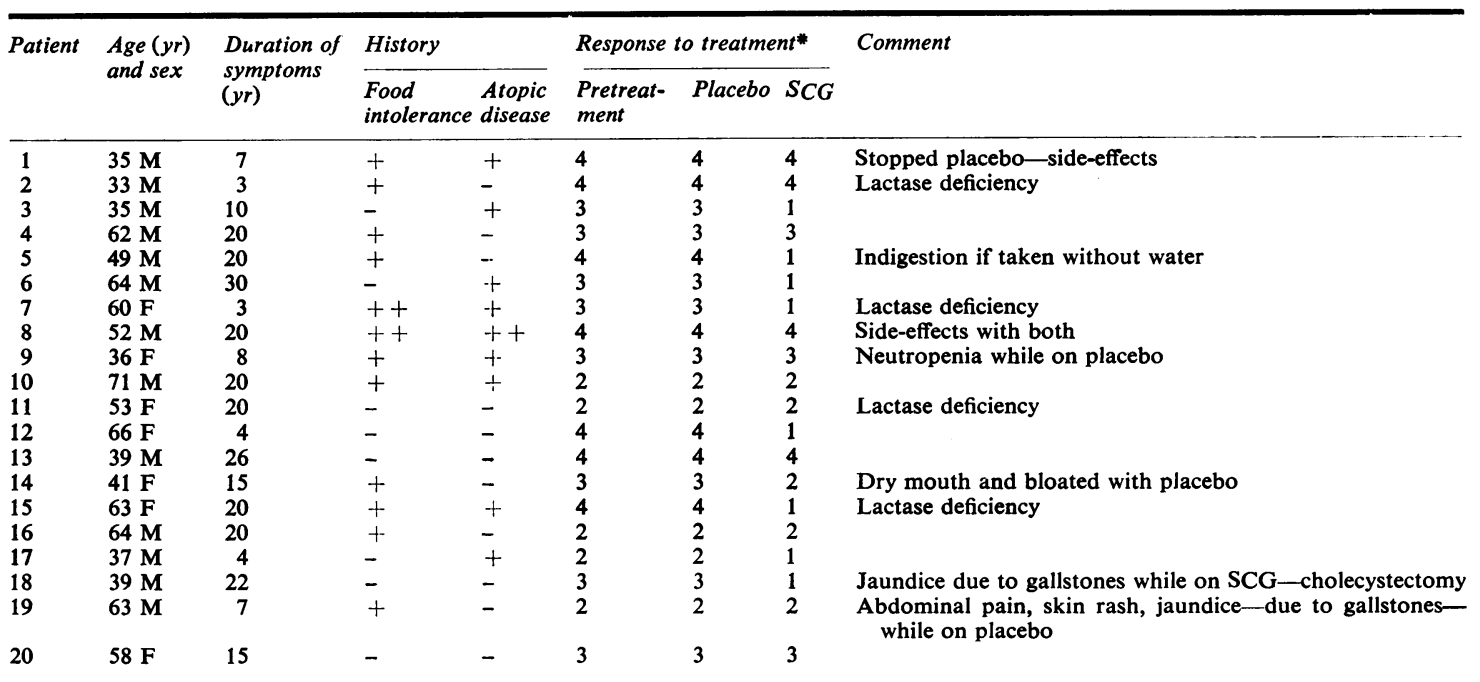

*Stools per day: 1: one-two formed. 2: one-two unformed. 3: three-four unformed. 4: more than four unformed.

Improvement with SCG was significant-0.05 $>$ P $>0.01$, McNemar's test (corrected for small numbers).

severity was made by both patient and clinician at the end of each treatment period. The dosage of oral SCG was $200 \mathrm{mg}$ four times daily. Placebo capsules contained an equivalent volume of glucose. Significant improvement was judged to have occurred if there was a reduction in frequency of stools by $50 \%$, associated with an increased firmness of consistency.

\section{Results (Table)}

Twenty patients completed the trial. Eight noted significant benefit from the use of oral SCG with a reduction in frequency and firmer consistency of their motions. Two patients who were socially disabled because of the severity and urgency of their diarrhoea dramatically improved on SCG and resumed their normal activities.

The remaining 12 patients found no benefit from either medication or, alternatively, improved with both (three patients). There was nothing to suggest continued activity of SCG into the placebo period.

The presence of a positive history of 'food intolerance' or of atopic disease bore no relationship to a beneficial response to SCG nor did a family history of atopic disease. Three responders described intolerance to food which was neither specific nor predictable; five responders had a history of rhinitis/ asthma but this did not help in predicting a beneficial response.

Lactase deficiency was present in four of the 12 patients who had a small bowel biopsy and this did not correlate with a response to SCG. In none was the jejunal histology abnormal.

Only one patient described side-effects attributed to SCG and this consisted of 'indigestion' when the drug was taken without water. Gallstones were noted during treatment in two patients; one on SCG and another on placebo. Three patients described side-effects while taking the placebo and neutropenia was detected in another patient on placebo. In this patient placebo therapy antedated that with SCG and neutropenia spontaneously returned to normal.

\section{Discussion}

The use of oral SCG has been described in food allergies $;^{4-8}$ these studies, however, were primarily directed towards a wide variety of allergic reactions including angio-oedema, rhinitis, cough, headache, and fatigue, rather than being primarily concerned with gastrointestinal symptoms. This present study was concerned solely with diarrhoea as a possible manifestation of allergic reactions of the gut to food in general. The finding of symptomatic improvement in $40 \%$ of the patients who had in the past tried a multitude of symptomatic measures, suggests that oral SCG has a role to play in treating persistent diarrhoea without an obvious organic cause. As no single food could be identified as the offending substance by patients who responded, oral SCG may be a simpler alternative to exclusion diets, provided 
due regard is paid to the need for tailoring the dose of SCG for individuals, depending on the severity and timing of their symptoms.

Fisons Pty. Limited kindly supplied the sodium cromoglycate.

\section{References}

${ }^{1}$ Anonymous. Management of the irritable bowel (Editorial). Lancet 1978; 2: 557-8.

${ }^{2}$ Brostoff J, Carini C, Wraith DG, Johns P. Production of IgE complexes by allergen challenge in atopic patients and the effect of sodium cromoglycate. Lancet 1979; 1: 1268-70.

${ }^{3}$ Paganelli R, Levinsky RJ, Brostoff J, Wraith DG. Immune complexes containing food proteins in normal and atopic subjects after oral challenge and effect of sodium cromoglycate on antigen absorption. Lancet $1979 ; 1$ : 1270-2.

${ }^{4} \mathrm{Vaz}$ GA, Tan LK-T, Gerrard JW. Oral cromoglycate in treatment of adverse reactions to foods. Lancet 1978; 1: 1066-8.

${ }^{5}$ Freier S, Berger H. Disodium cromoglycate in gastrointestinal protein intolerance. Lancet 1973; 1: 913-5.

${ }^{6}$ Kuzemko JA, Simpson KR. Treatment of allergy to cow's milk. (Letter) Lancet 1975; 1 : 337-8.

${ }^{7}$ Dannaeus A, Foucard T, Johannson SGO. The effect of orally administered sodium cromoglycate on symptoms of food allergy. Clinical Allergy 1977; 7: 109-15.

${ }^{8}$ Nizami RM, Lewin PK, Baboo MT. Oral Cromolyn therapy in patients with food allergy: a preliminary report. Ann Allergy 1977; 39: 102-5. 